\title{
Skewness and kurtosis of net baryon-number distributions at small values of the baryon chemical potential
}

\author{
A. Bazavov, ${ }^{1}$ H.-T. Ding, ${ }^{2}$ P. Hegde, ${ }^{3}$ O. Kaczmarek, ${ }^{4}$ F. Karsch,,${ }^{4,5}$ E. Laermann, ${ }^{4}$ Swagato Mukherjee, ${ }^{5}$ \\ H. Ohno, ${ }^{5,6}$ P. Petreczky, ${ }^{5}$ E. Rinaldi, ${ }^{7}$ H. Sandmeyer, ${ }^{4}$ C. Schmidt, ${ }^{4}$ Chris Schroeder, ${ }^{8}$ S. Sharma, ${ }^{5}$ \\ W. Soeldner, ${ }^{9}$ R. A. Soltz, ${ }^{8}$ P. Steinbrecher, ${ }^{4,5}$ and P. M. Vranas ${ }^{8}$
}

(HotQCD Collaboration)

\author{
${ }^{1}$ Department of Computational Mathematics, Science and Engineering and Department of Physics \\ and Astronomy, Michigan State University, East Lansing, Michigan 48824, USA \\ ${ }^{2}$ Key Laboratory of Quark \& Lepton Physics (MOE) and Institute of Particle Physics, Central China \\ Normal University, Wuhan 430079, China \\ ${ }^{3}$ Center for High Energy Physics, Indian Institute of Science, Bangalore 560012, India \\ ${ }^{4}$ Fakultät für Physik, Universität Bielefeld, D-33615 Bielefeld, Germany \\ ${ }^{5}$ Physics Department, Brookhaven National Laboratory, Upton, New York 11973, USA \\ ${ }^{6}$ Center for Computational Sciences, University of Tsukuba, Tsukuba, Ibaraki 305-8577, Japan \\ ${ }^{7}$ RIKEN-BNL Research Center, Brookhaven National Laboratory, Upton, New York 11973, USA \\ ${ }^{8}$ Physics Division, Lawrence Livermore National Laboratory, Livermore, California 94550, USA \\ ${ }^{9}$ Institut für Theoretische Physik, Universität Regensburg, D-93040 Regensburg, Germany
}

(Received 20 August 2017; published 27 October 2017)

\begin{abstract}
We present results for the ratios of mean $\left(M_{B}\right)$, variance $\left(\sigma_{B}^{2}\right)$, skewness $\left(S_{B}\right)$ and kurtosis $\left(\kappa_{B}\right)$ of net baryon-number fluctuations obtained in lattice QCD calculations with physical values of light and strange quark masses. Using next-to-leading order Taylor expansions in baryon chemical potential we find that qualitative features of these ratios closely resemble the corresponding experimentally measured cumulant ratios of net proton-number fluctuations for beam energies down to $\sqrt{s_{N N}} \geq 19.6 \mathrm{GeV}$. We show that the difference in cumulant ratios for the mean net baryon-number, $M_{B} / \sigma_{B}^{2}=\chi_{1}^{B}\left(T, \mu_{B}\right) / \chi_{2}^{B}\left(T, \mu_{B}\right)$, and the normalized skewness, $S_{B} \sigma_{B}=\chi_{3}^{B}\left(T, \mu_{B}\right) / \chi_{2}^{B}\left(T, \mu_{B}\right)$, naturally arises in QCD thermodynamics. Moreover, we establish a close relation between skewness and kurtosis ratios, $S_{B} \sigma_{B}^{3} / M_{B}=\chi_{3}^{B}\left(T, \mu_{B}\right) / \chi_{1}^{B}\left(T, \mu_{B}\right)$ and $\kappa_{B} \sigma_{B}^{2}=\chi_{4}^{B}\left(T, \mu_{B}\right) / \chi_{2}^{B}\left(T, \mu_{B}\right)$, valid at small values of the baryon chemical potential.
\end{abstract}

DOI: $10.1103 /$ PhysRevD.96.074510

\section{INTRODUCTION}

Fluctuations of [1,2] and correlations among [3] conserved charges of strong interactions have long been considered as sensitive observables to explore the structure of the phase diagram of Quantum Chromodynamics (QCD). These are accessible to lattice QCD calculations [4] and are also the most promising observables in the experimental search for the conjectured critical point $[5,6]$ in the phase diagram of QCD that is currently underway with the beam energy scan (BES) program at the Relativistic Heavy Ion Collider (RHIC) [7]. The results on net electric charge [8,9] and net proton-number [10-12] fluctuations obtained from the first BES runs at RHIC have not yet provided conclusive evidence for the existence of a critical point. However, the data on the skewness and kurtosis of the distribution of net proton-number fluctuations show an intriguing dependence on the beam energy. The published data on cumulants of net proton-number fluctuations [10] and, in particular, the preliminary data set on net proton-number fluctuations measured in an extended transverse momentum range [11,12] show obvious deviations from the thermodynamics of a hadron resonance gas (HRG). The ratios of even order cumulants, as well as the ratios of odd order cumulants, differ from unity, and different mixed ratios formed from even and odd order cumulants are not identical. This may not be too surprising as HRG model calculations are not expected to give an accurate description of the thermodynamics of strong interaction matter, described by QCD. However, these experimental findings raise the question whether the observed pattern seen in net proton-number fluctuations can be understood in terms of QCD thermodynamics, which provides information on net baryon-number fluctuations in equilibrium [13], or whether other effects such as acceptance cuts, limited efficiencies, and rapidity dependence [14-18], or nonequilibrium effects [19-22], are responsible for these differences (for a recent review see [23]).

At large beam energies net proton-number densities are small and the baryon chemical potential $\left(\mu_{B}\right)$ is close to zero, e.g. $\mu_{B} / T \simeq 0.2$ at $\sqrt{s_{N N}}=200 \mathrm{GeV}$. It is, thus, 
conceivable that low order Taylor expansions are also suitable for the description of the cumulants of the net baryon-number fluctuations at the time of freeze-out [24]. In fact, the calculations of various thermodynamic observables in a Taylor series in baryon chemical potential suggest that expansions up to next-to-leading order (NLO) in $\mu_{B}$ provide good approximations for these observables for $\mu_{B} / T \lesssim(1.5-2)$ [25]. In the transition region, characterized by the pseudocritical temperature for the chiral transition, $T_{c}=154(9) \mathrm{MeV}$ [26], one thus may expect to obtain reliable results up to baryon chemical potentials $\mu_{B} \simeq$ (225-300) MeV within NLO Taylor expansion. Comparison of cumulant ratios of net electric charge fluctuations, measured at various beam energies, with lattice QCD results [27-30] as well as HRG model calculations $[8,31,32]$ suggests that $\mu_{B}<1.4 T$ for $\sqrt{s_{N N}} \geq 19.6 \mathrm{GeV}$. NLO Taylor expansions of cumulants of conserved charge fluctuations, thus, should provide an adequate description of equilibrium thermodynamics of strong interaction matter for a large part of the beam energies probed with the BES at RHIC, $7.7 \mathrm{GeV} \leq \sqrt{s_{N N}} \leq 200 \mathrm{GeV}$.

The purpose of this paper is to determine, within the framework of equilibrium thermodynamics of QCD, the dependence of net baryon-number fluctuations on temperature and baryon chemical potential along lines in the $T-\mu_{B}$ plane. We will focus on an analysis of thermodynamic properties of ratios of cumulants formed from the first four cumulants of net baryon-number fluctuations, i.e. mean $\left(M_{B}\right)$, variance $\left(\sigma_{B}^{2}\right)$, skewness $\left(S_{B}\right)$, and kurtosis $\left(\kappa_{B}\right)$ of net baryon-number distributions,

$$
\begin{aligned}
& R_{12}^{B}\left(T, \mu_{B}\right) \equiv \frac{\chi_{1}^{B}\left(T, \mu_{B}\right)}{\chi_{2}^{B}\left(T, \mu_{B}\right)} \equiv \frac{M_{B}}{\sigma_{B}^{2}}, \\
& R_{31}^{B}\left(T, \mu_{B}\right) \equiv \frac{\chi_{3}^{B}\left(T, \mu_{B}\right)}{\chi_{1}^{B}\left(T, \mu_{B}\right)} \equiv \frac{S_{B} \sigma_{B}^{3}}{M_{B}}, \\
& R_{42}^{B}\left(T, \mu_{B}\right) \equiv \frac{\chi_{4}^{B}\left(T, \mu_{B}\right)}{\chi_{2}^{B}\left(T, \mu_{B}\right)} \equiv \kappa_{B} \sigma_{B}^{2} .
\end{aligned}
$$

Here the $n$-th order cumulants, $\chi_{n}^{B}\left(T, \mu_{B}\right)$, are obtained from partial derivatives of the QCD pressure, $P\left(T, \mu_{B}, \mu_{Q}, \mu_{S}\right)$, with respect to the baryon chemical potential $\mu_{B}$. Obviously, the ratio $R_{32}^{B} \equiv S_{B} \sigma_{B}$, which also is considered frequently as an experimental observable, is simply obtained from the above three independent ratios,

$$
R_{32}^{B}\left(T, \mu_{B}\right)=R_{31}^{B} R_{12}^{B}=\frac{\chi_{3}^{B}\left(T, \mu_{B}\right)}{\chi_{2}^{B}\left(T, \mu_{B}\right)} \equiv S_{B} \sigma_{B} .
$$

We will provide lattice QCD results on cumulants of conserved charge fluctuations in next-to-leading order Taylor expansions. We will confront these results with experimental data on cumulants of net proton-number fluctuations $\left(M_{P}, \sigma_{P}^{2}, S_{P}, \kappa_{P}\right)$ [10,11], obtained by the STAR collaboration during the first BES at RHIC.
Already at large beam energies, i.e. small values of the baryon chemical potential, these data significantly deviate from expectations based on HRG model calculations, which are commonly used as a baseline for the analysis of data on particle yields and charge fluctuations in terms of equilibrium thermodynamics [33]. In particular data suggest, that

(i) The ratio $M_{P} / \sigma_{P}^{2}$ is a monotonically decreasing function of $\sqrt{s_{N N}}$, and $M_{P} / \sigma_{P}^{2}>S_{P} \sigma_{P}$ in the entire range of $\sqrt{s_{N N}}$ probed so far.

(ii) $S_{P} \sigma_{P}^{3} / M_{P}$ is smaller than unity and has a weak but significant dependence on $\sqrt{s_{N N}}$ becoming increasingly smaller than unity with decreasing $\sqrt{s_{N N}}$ or, equivalently, with increasing $M_{P} / \sigma_{P}^{2}$.

(iii) For $M_{P} / \sigma_{P}^{2}=0$ or, equivalently, for large $\sqrt{s_{N N}}$, the relation $S_{P} \sigma_{P}^{3} / M_{P} \simeq \kappa_{P} \sigma_{P}^{2}$ seems to hold quite well even though both ratios individually remain smaller than unity.

(iv) With decreasing $\sqrt{s_{N N}}$ or, equivalently, increasing $M_{P} / \sigma_{P}^{2}$, the cumulant ratio $\kappa_{P} \sigma_{P}^{2}$ departs further away from unity than the skewness ratio $S_{P} \sigma_{P}^{3} / M_{P}$. It seems that the inequality $\kappa_{P} \sigma_{P}^{2}<S_{P} \sigma_{P}^{3} / M_{P}<1$ holds at least for all beam energies $\sqrt{s_{N N}} \geq$ $19.6 \mathrm{GeV}$.

We will show here that the QCD calculations of net baryon-number fluctuations up to NLO in $\mu_{B} / T$ show all the above qualitative features of the cumulants of net proton-number fluctuations.

The paper is organized as follows: in Sec. II we introduce the basic expressions for the NLO expansions of cumulants of conserved charge fluctuations. In particular, we will derive the formulas needed to describe the variation of ratios of cumulants on a line in the $T-\mu_{B}$ plane of the QCD phase diagram. Details of our lattice QCD calculations are described in Sec. III. In Sec. IV and Sec. V we present results on the LO and NLO Taylor coefficients of cumulant ratios as function of $\mu_{B}$. We compare these NLO lattice QCD results on net baryon-number fluctuations to experimental data on net proton-number fluctuations in Sec. VI. Finally, we summarize the QCD results on the next-toleading order expansion of cumulant ratios and give some conclusions in Sec. VII. Further details on the NLO expansion coefficients are presented in the Appendix.

\section{TAYLOR EXPANSIONS OF CUMULANTS OF CONSERVED CHARGE FLUCTUATIONS}

\section{A. Expansions at fixed temperature}

For small values of the baryon chemical potential the cumulants of net baryon-number $(B)$, net electric charge $(Q)$, or net strangeness $(S)$ fluctuations,

$$
\chi_{n}^{X}(T, \vec{\mu})=\frac{\partial^{n} P / T^{4}}{\partial\left(\mu_{X} / T\right)^{n}}, \quad X=B, Q, S,
$$


with $\vec{\mu}=\left(\mu_{B}, \mu_{Q}, \mu_{S}\right)$, are easily obtained from a Taylor expansion of the QCD pressure. Using $\hat{\mu}_{X} \equiv \mu_{X} / T$ the pressure is given by

$$
\frac{P(T, \vec{\mu})}{T^{4}}=\sum_{i, j, k=0}^{\infty} \frac{1}{i ! j ! k !} \chi_{i j k}^{B Q S}(T) \hat{\mu}_{B}^{i} \hat{\mu}_{Q}^{j} \hat{\mu}_{S}^{k},
$$

where the expansion coefficients $\chi_{i j k}^{B Q S}$ are generalized susceptibilities,

$$
\chi_{i j k}^{B Q S}(T)=\left.\frac{\partial^{(i+j+k)} P / T^{4}}{\partial \hat{\mu}_{B}^{i} \partial \hat{\mu}_{Q}^{j} \partial \hat{\mu}_{S}^{k}}\right|_{\vec{\mu}=0},
$$

which can be evaluated in lattice QCD calculations performed at vanishing chemical potential. They are functions of the temperature, which we usually will not mention explicitly, $\chi_{i j k}^{B Q S} \equiv \chi_{i j k}^{B Q S}(T)$. We give the arguments only for the nonexpanded cumulants which are functions of $T$ as well as $\vec{\mu}$, i.e. $\chi_{n}^{X}(T, \vec{\mu})$. In the following we will also adopt the convention to suppress subscripts and superscripts of the expansion coefficients whenever a subscript vanishes, e.g. $\chi_{101}^{B Q S} \equiv \chi_{11}^{B S}$ etc.

We will focus on NLO expansions of the first four cumulants along a line in the space of the three chemical potentials. This line is fixed by two constraints which relate the electric charge and strangeness chemical potentials to the baryon chemical potential [28]. In NLO one may parametrize them as

$$
\begin{aligned}
\hat{\mu}_{Q}\left(T, \mu_{B}\right) & =q_{1}(T) \hat{\mu}_{B}+q_{3}(T) \hat{\mu}_{B}^{3}+\mathcal{O}\left(\hat{\mu}_{B}^{5}\right), \\
\hat{\mu}_{S}\left(T, \mu_{B}\right) & =s_{1}(T) \hat{\mu}_{B}+s_{3}(T) \hat{\mu}_{B}^{3}+\mathcal{O}\left(\hat{\mu}_{B}^{5}\right) .
\end{aligned}
$$

In applications to heavy ion collisions it is appropriate to determine the coefficients $q_{i}, s_{i}$ from constraints demanding overall vanishing net strangeness density,

$$
n_{S} \equiv \chi_{1}^{S}(T, \vec{\mu})=0,
$$

and a fixed relation between net baryon-number and net electric charge densities,

$$
\frac{n_{Q}}{n_{B}} \equiv \frac{\chi_{1}^{Q}(T, \vec{\mu})}{\chi_{1}^{B}(T, \vec{\mu})}=\frac{N_{P}}{N_{P}+N_{N}} .
$$

Here the last equality relates the ratio of the number densities to the proton $\left(N_{P}\right)$ and neutron $\left(N_{N}\right)$ numbers of the incident nuclei in heavy ion collision experiments. In the case of gold or uranium nuclei, which are frequently used in heavy ion collision experiments, setting $n_{Q} / n_{B}=$ 0.4 is appropriate [28]. The isospin symmetric case obviously corresponds to $n_{Q} / n_{B}=1 / 2$. In that case $\mu_{Q}=$ 0 and, thus, $q_{i}=0$ at all orders. Explicit expressions for $q_{i}$, $s_{i}$ have been given in Appendix B of Ref. [25].
We will discuss Taylor expansions for the ratios of cumulants introduced in Eq. (1) and Eq. (2). Using the parametrization of $\mu_{Q}$ and $\mu_{S}$ given in Eq. (6), we may write these expansions in terms of $\hat{\mu}_{B}$ up to NLO,

$$
\begin{gathered}
R_{12}^{B}\left(T, \mu_{B}\right)=r_{12}^{B, 1} \hat{\mu}_{B}+r_{12}^{B, 3} \hat{\mu}_{B}^{3}, \\
R_{31}^{B}\left(T, \mu_{B}\right)=r_{31}^{B, 0}+r_{31}^{B, 2} \hat{\mu}_{B}^{2}, \\
R_{42}^{B}\left(T, \mu_{B}\right)=r_{42}^{B, 0}+r_{42}^{B, 2} \hat{\mu}_{B}^{2} .
\end{gathered}
$$

Here the expansion coefficients $r_{n m}^{B, k}$ are functions of temperature and the Taylor expansion coefficients $q_{i}, s_{i}$ of the constraint chemical potentials $\mu_{Q}, \mu_{S}$. The superscript $k$ labels the order of the expansion in terms of $\mu_{B}$. Explicit expressions for the expansion coefficients $r_{n m}^{B, k}$ in terms of the generalized susceptibilities, introduced in Eq. (5), are given in the Appendix.

\section{B. Expansions along lines $T\left(\mu_{B}\right)$ in the $T-\mu_{B}$ plane}

It is our goal to compare cumulant ratios measured in heavy ion experiments at different beam energies, $\sqrt{s_{N N}}$, with lattice QCD calculations of such ratios. As the beam energy is varied also the thermal conditions under which particles "freeze-out" change. This is commonly characterized by a pair of freeze-out parameters $\left(T_{f}, \mu_{B}\right)$. They map out a line, $T_{f}\left(\mu_{B}\right)$, in the QCD phase diagram. When comparing the Taylor expanded cumulant ratios, discussed in the previous subsection, with experimental data we thus also need to take into account that the freeze-out temperature varies with increasing $\mu_{B}$. At large beam energies it is appropriate to parametrize the freeze-out line as a polynomial in $\mu_{B}^{2}$ [31]. ${ }^{1}$

In the context of Taylor expansions for bulk thermodynamic observables, "lines of constant physics" [25] as well as the pseudocritical line for the QCD transition [34-37] are generally given as polynomials in $\mu_{B}^{2}$. We thus will consider the behavior of cumulants of conserved charge fluctuations on lines in the $T-\mu_{B}$ plane that are parametrized as

$$
T_{f}\left(\mu_{B}\right)=T_{0}\left(1-\kappa_{2}^{f} \bar{\mu}_{B}^{2}+\mathcal{O}\left(\bar{\mu}_{B}^{4}\right)\right),
$$

with $\bar{\mu}_{B} \equiv \mu_{B} / T_{0}$. As we will exploit only NLO expansions for cumulants it suffices to know this parametrization up to $\mathcal{O}\left(\mu_{B}^{2}\right)$.

\footnotetext{
${ }^{1}$ Such a parametrization is commonly used when comparing experimental data on particle yields with statistical hadronization models (HRG models). An alternative parametrization, used in Ref. [32], also provides a good description of the experimental data but does not have a polynomial behavior for small $\mu_{B}$. It starts out with exponentially small corrections to the freeze-out temperature at vanishing $\mu_{B}$.
} 
Taking into account this temperature variation requires an additional expansion of the ratios $R_{n m}^{B}$ in $T$. On a line $T_{f}\left(\mu_{B}\right)$ the Taylor expansion in $T$ then generates additional terms that are of order $\mu_{B}^{2}$. I.e., the LO expansion coefficients of cumulant ratios remain unchanged, while the NLO expansion coefficients, $r_{n m}^{B, k}(T)$, receive an additional contribution from the variation of cumulant ratios with temperature along a line in the $T-\mu_{B}$ plane,

$$
r_{n m}^{B, k} \rightarrow r_{n m, f}^{B, k} \equiv r_{n m}^{B, k}\left(T_{0}\right)-\left.\kappa_{2}^{f} T_{0} \frac{\mathrm{d} r_{n m}^{B, k-2}}{\mathrm{~d} T}\right|_{T=T_{0}},
$$

with $k=2$ or 3 . With this, the three cumulant ratios introduced in Eq. (1) become

$$
\begin{gathered}
R_{12}^{B}\left(T_{f}\left(\mu_{B}\right), \mu_{B}\right)=r_{12}^{B, 1} \bar{\mu}_{B}+r_{12, f}^{B, 3} \bar{\mu}_{B}^{3}, \\
R_{31}^{B}\left(T_{f}\left(\mu_{B}\right), \mu_{B}\right)=r_{31}^{B, 0}+r_{31, f}^{B, 2} \bar{\mu}_{B}^{2}, \\
R_{42}^{B}\left(T_{f}\left(\mu_{B}\right), \mu_{B}\right)=r_{42}^{B, 0}+r_{42, f}^{B, 2} \bar{\mu}_{B}^{2} .
\end{gathered}
$$

Here all expansion coefficients $r_{n m}^{B, k}$ and $r_{n m, f}^{B, k}$ are evaluated at $\mu_{B}=0$ and at the temperature $T\left(\mu_{B}=0\right) \equiv T_{0}$.

In the following sections we will present lattice QCD results for the expansion coefficients appearing in Eqs. (14)-(16). In particular, as done before in an analysis of ratios of variances of net electric charge and net baryonnumber fluctuations [38], we will make use of the fact that $r_{12}^{B, 1}$ is positive for all values of the temperature. At least to leading order in $\mu_{B}$ the ratio $M_{B} / \sigma_{B}^{2}$, thus, is a monotonically rising function of $\mu_{B}$. We may use this to eliminate the baryon chemical potential $\mu_{B}$ in the NLO relations for $R_{31}^{B}$ and $R_{42}^{B}$ in favor of the mean net baryon-number ratio, $R_{12}^{B} \equiv M_{B} / \sigma_{B}^{2}$, i.e.

$$
\hat{\mu}_{B}=\frac{1}{r_{12}^{B, 1}} R_{12}^{B}+\mathcal{O}\left(\left(R_{12}^{B}\right)^{3}\right) .
$$

With this we obtain at NLO

$$
\begin{aligned}
& R_{31}^{B}\left(T, R_{12}^{B}\right)=r_{31}^{B, 0}+\frac{r_{31, f}^{B, 2}}{\left(r_{12}^{B, 1}\right)^{2}}\left(R_{12}^{B}\right)^{2}, \\
& R_{42}^{B}\left(T, R_{12}^{B}\right)=r_{42}^{B, 0}+\frac{r_{42, f}^{B, 2}}{\left(r_{12}^{B, 1}\right)^{2}}\left(R_{12}^{B}\right)^{2} .
\end{aligned}
$$

Using Eq. (2) we easily obtain from Eq. (18), also, the NLO expansion for the ratio $R_{32}^{B}$,

$$
R_{32}^{B}\left(T, R_{12}^{B}\right)=r_{31}^{B, 0} R_{12}^{B}+\frac{r_{31, f}^{B, 2}}{\left(r_{12}^{B, 1}\right)^{2}}\left(R_{12}^{B}\right)^{3} .
$$

Considering expansions of higher order cumulant ratios in terms of the lowest order ratio $R_{12}^{B}$ rather than expansions in $\hat{\mu}_{B}$ has the advantage that we can compare the QCD results directly to experimental observables without the need of first determining a chemical potential from the data. A trivial consequence is that at LO the slope of the expansion of $R_{32}^{B}$ in terms of $R_{12}^{B}$ is identical to the intercept of $R_{31}^{B}$ at $\mu_{B}=0$.

We note that in the low temperature HRG limit $R_{12}^{B} \simeq \tanh \hat{\mu}_{B}$, which can be inverted for all $\hat{\mu}_{B}$. However, in the vicinity of a possible critical point in the $T-\mu_{B}$ plane $R_{12}^{B}$ will no longer be a monotonic function of $\mu_{B}$ as $\sigma_{B}^{2}$ will diverge at a critical point while $M_{B}$ stays finite. In the parameter range probed experimentally so far, no indication for such a nonmonotonic behavior of $R_{12}^{B}$ has been observed.

\section{LATTICE QCD CALCULATIONS}

The main results presented in the following are based on lattice QCD calculations performed in the temperature range $135 \mathrm{MeV} \lesssim T \lesssim 175 \mathrm{MeV}$. In this temperature interval our analysis is based on calculations performed with a strange quark mass tuned to its physical value and degenerate light quarks with a mass $m_{l} / m_{s}=1 / 27$. In the continuum limit this light quark mass corresponds to a pion mass of about $140 \mathrm{MeV}$. For completeness and in order to give a feeling for the trends in the temperature dependence of various observables, we added a few data at higher $T$-values that have been obtained from calculations with a somewhat larger quark mass ratio, $m_{l} / m_{s}=1 / 20$. In the continuum limit this quark mass ratio corresponds to a pion mass of about $160 \mathrm{MeV}$.

The parameter choices, lattice sizes, quark masses, as well as the determination of the temperature scale from zero temperature observables, are identical to the setup used previously in our calculation of the equation of state at vanishing chemical potential [39] and the calculation of the equation of state of $(2+1)$-flavor QCD at nonzero baryon chemical potential in 6th order Taylor series [25].

Our calculations are performed on lattices of size $N_{\sigma}^{3} \times N_{\tau}$ with $N_{\sigma}=4 N_{\tau}$ and $N_{\tau}=6,8,12,16$. Compared to earlier calculations [25] we have increased the statistics on the $32^{3} \times 8$ and $48^{3} \times 12$ lattices by about a factor of four and added new calculations on lattices of size $64^{3} \times 16$.

Our main conclusions on the behavior of NLO expansion coefficients are based on calculations performed on lattices of size $32^{3} \times 8$, where we generated up to 700,000 gauge field configurations using the Rational Hybrid Monte Carlo (RHMC) algorithm. We generated up to 7 million RHMC trajectories of unit length and saved gauge field configurations after every 10th trajectory. Our current statistics are summarized in Table I.

Up to 6th order cumulants have been calculated on these data sets. Due to the large number of gauge field 
TABLE I. Number of gauge field configurations on lattices of size $32^{3} \times 8,48^{3} \times 12$, and $64^{3} \times 16$ used in the analysis of up to 6th order Taylor expansion coefficients. The values of the gauge coupling as well as the strange and light quark mass parameter at these temperature values are taken from [25], where also details on the statistics available on the $24^{3} \times 6$ lattices are given.

\begin{tabular}{lccccc}
\hline \hline & $N_{\tau}=8$ & \multicolumn{2}{c}{$N_{\tau}=12$} & $N_{\tau}=16$ \\
$T[\mathrm{MeV}]$ & Number of configurations & $T[\mathrm{MeV}]$ & Number of configurations & $T[\mathrm{MeV}]$ & Number of configurations \\
\hline 134.64 & 456070 & 134.94 & 39380 & $\ldots$ & \\
140.45 & 626790 & 140.44 & 61610 & $\ldots$ & 2980 \\
144.95 & 684200 & 144.97 & 69910 & 144.94 & 8080 \\
151.00 & 362200 & 151.10 & 45900 & 151.04 & 4850 \\
156.78 & 513130 & 157.13 & 30100 & 156.92 & 2510 \\
162.25 & 247040 & 161.94 & 32810 & 162.10 & 2430 \\
165.98 & 283640 & 165.91 & 64820 & 166.03 & 170.98 \\
171.02 & 139980 & 170.77 & 40870 & $\ldots$ & \\
175.64 & 137500 & 175.77 & 39040 & & \\
\hline \hline
\end{tabular}

configurations needed for an analysis of the expansion coefficients of kurtosis and skewness ratios, our main results on NLO expansion coefficients for these observables are based on calculations for a single lattice size only; i.e., they are not yet continuum extrapolated, although cutoff effects are expected to be significantly smaller in theses observables than our current statistical errors.

For the LO observables, continuum extrapolations were done using global spline fits to data from all 4 lattice spacings following the procedure described in $[25,38]$. We allow for $1 / N_{\tau}^{2}$ dependence of the spline coefficients and also vary the locations of the spline knots to minimize the $\chi^{2}$ of the global fits. For the current analysis we found it is sufficient to use spline interpolations with quartic polynomials and 3 knots whose location is allowed to vary in the fit range. Fits were done for many bootstrap samples drawn from the Gaussian errors of data points, and final results were obtained from mean values and standard deviations of the bootstrapped fit results, weighted by the quality of the fits given by the Akaike information criteria. For the NLO observables, we have lattice data only for 2 lattice spacings corresponding to $N_{\tau}=6$ and 8 , and we could not carry out proper continuum extrapolations. For these cases, we provide "continuum estimates" following exactly the same continuum extrapolation procedure described above, but only using global spline fits to the data from $N_{\tau}=6$ and 8 lattices.

In the following three sections we will present results on the various $\mathrm{LO}$ and NLO expansion coefficients entering in Eqs. (17)-(20).

\section{LEADING ORDER EXPANSION OF CUMULANT RATIOS}

The leading order expansion coefficients for the ratios $R_{n m}^{B}$ defined in Eq. (1) are given in the Appendix. We can write them as

$$
\begin{gathered}
r_{12}^{B, 1}=1+s_{1} \frac{\chi_{11}^{B S}}{\chi_{2}^{B}}+q_{1} \frac{\chi_{11}^{B Q}}{\chi_{2}^{B}}, \\
r_{42}^{B, 0}=\frac{\chi_{4}^{B}}{\chi_{2}^{B}}, \\
r_{31}^{B, 0}=r_{42}^{B, 0} \frac{1+s_{1} \frac{\chi_{31}^{B S}}{\chi_{4}^{B}}+q_{1} \frac{\chi_{31}^{B Q}}{1+s_{1} \frac{\chi_{11}^{B S}}{\chi_{2}^{B}}+q_{1} \frac{\chi_{11}^{B Q}}{\chi_{2}^{B}}} .}{}
\end{gathered}
$$

This makes it apparent that the LO coefficients are particularly simple for $\mu_{S}=\mu_{Q}=0$. In that case, one has $r_{12}^{B, 1}=1$ and $r_{31}^{B, 0}=r_{42}^{B, 0}$. In the strangeness neutral case with fixed $n_{Q} / n_{B}=0.4$ the contribution from a nonvanishing electric charge chemical potential is small. The dominant correction arises from a nonzero strangeness chemical potential needed to insure strangeness neutrality [40]. As $s_{1}>0$ and $\chi_{11}^{B S} / \chi_{2}^{B}<0$ we thus expect to find $r_{12}^{B, 1}<1$. This is also the case at low temperature for a HRG.

In Fig. 1 we show results for the leading order expansion coefficients of the ratios $R_{12}^{B}, R_{31}^{B}$, and $R_{42}^{B}$, respectively. The left-hand figure shows the LO expansion coefficient $r_{12}^{B, 1}$. This is an update on results obtained previously in [38] from calculations with much lower statistics. The righthand part of the figure shows the LO result $r_{42}^{B, 0}$. Also shown as an inset in this figure is the difference between the leading order results for $r_{42}^{B, 0}$ and $r_{31}^{B, 0}$, normalized to the latter. The LO results for the cumulant ratios shown in this figure have been obtained for a strangeness neutral system, $n_{S}=0$, with electric charge to baryon-number ratio $n_{Q} / n_{B}=0.4$.

Let us first discuss the leading order results for the ratio $R_{12}^{B}\left(T, \mu_{B}\right)$. Here results from calculations on lattices with temporal extent $N_{\tau}=6$ to 16 exist. They show rather small cutoff dependence, which is known also from our 

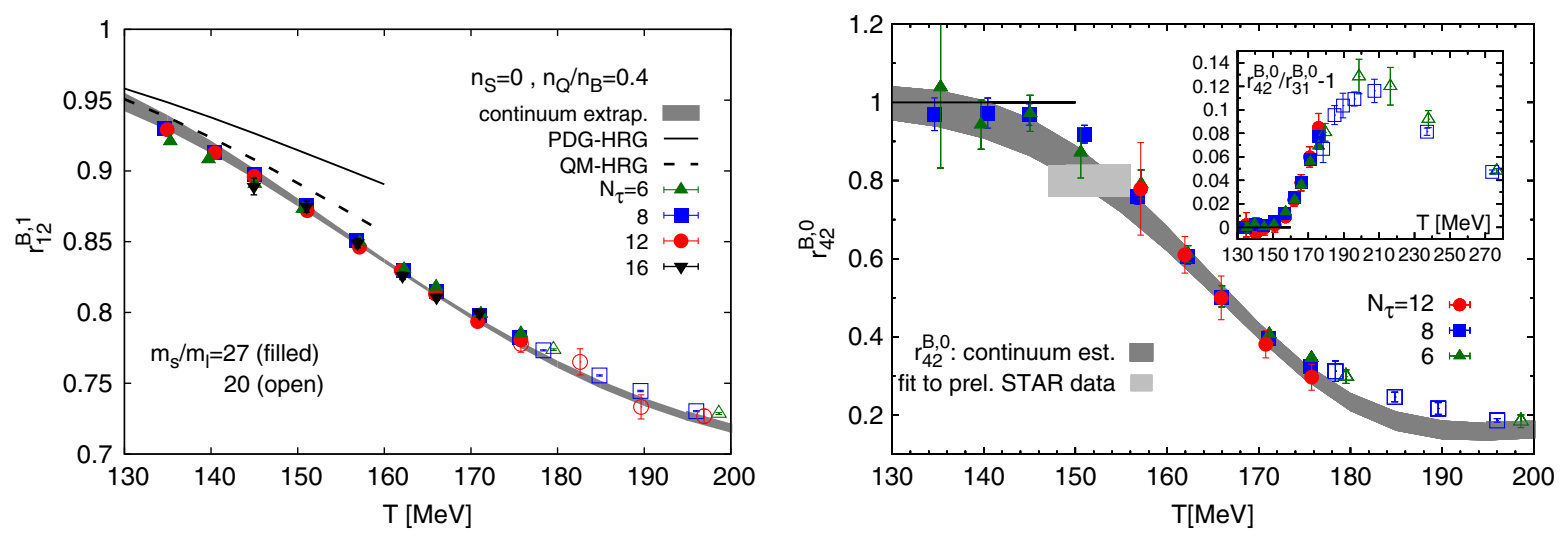

FIG. 1. The leading order expansion coefficients of the cumulant ratios $R_{12}^{B}$ (left) and $R_{42}^{B}$ (right) versus temperature calculated on lattices with temporal extent $N_{\tau}$, and spatial sizes $N_{\sigma}=4 N_{\tau}$. The inset in the right-hand figure shows the difference between the leading order results for the kurtosis ratio $R_{42}^{B}$ and the skewness ratio $R_{31}^{B}$ normalized to the latter. All expansion coefficients have been calculated for strangeness neutral systems, Eq. (7), with an electric charge to baryon-number ratio $r=0.4$ (Eq. (8). The gray bands give the continuum extrapolated result for $r_{12}^{B, 1}$ and, in the case of $r_{42}^{B, 0}$, an estimate for the continuum result. In the right-hand figure we also show results from a fit to the preliminary STAR data for the corresponding net proton-number fluctuations discussed in Sec. VI. See text for a discussion of the two HRG curves shown in the left-hand figure.

calculations of the Taylor expansion coefficients of the equation of state. The results have been extrapolated to the continuum limit using spline interpolations as described in Ref. [39]. Obviously $r_{12}^{B, 1}$ approaches the HRG value from below at all values of the temperature. As has been observed previously in calculations of cumulants that are sensitive to the strange baryon sector of hadron resonance gas models [40], we find that a HRG model, which includes additional strange baryons (QM-HRG) provides a better description of the Taylor expansion coefficients than a HRG model based only on experimentally well established resonances listed in the Particle Data Tables (PDG-HRG) [41].

Similarly, the LO expansion coefficients of the ratios $R_{31}^{B} \equiv S_{B} \sigma_{B}^{3} / M_{B}=r_{31}^{B, 0}+\mathcal{O}\left(\mu_{B}^{2}\right)$ and $R_{42}^{B} \equiv \kappa_{B} \sigma_{B}^{2}=r_{42}^{B, 0}+$ $\mathcal{O}\left(\mu_{B}^{2}\right)$, shown in Fig. 1 (right), seem to approach the HRG model value from below. At least for $T>150 \mathrm{MeV}$ these ratios are smaller than unity. As a consequence we find to LO in $\mu_{B}$, or equivalently to $\mathrm{LO}$ in $R_{12}^{B}$, that

$$
R_{32}^{B} \equiv R_{31}^{B} R_{12}^{B}<R_{12}^{B}+\mathcal{O}\left(\left(R_{12}^{B}\right)^{3}\right) .
$$

At least for $T>150 \mathrm{MeV}$ ratios of cumulants thus obey the inequality $M_{B} / \sigma_{B}^{2}>S_{B} \sigma_{B}$, or equivalently $R_{31}^{B} \equiv$ $S_{B} \sigma_{B}^{3} / M_{B}<1$. This clearly is different from HRG model calculations with pointlike noninteracting hadrons, where the exact relations, $M_{B} / \sigma_{B}^{2}=S_{B} \sigma_{B}$ and $S_{B} \sigma_{B}^{3} / M_{B}=1$, hold at any order in $\mu_{B}$, irrespective of the details of the hadron spectrum used in that calculation.

From the LO expressions given in Eq. (22) and Eq. (23), it is obvious that to leading order the ratios $R_{31}^{B}$ and $R_{42}^{B}$ will also be identical in the case of vanishing strangeness and electric charge chemical potentials, although their values need not be unity. Fig. 1 (right) shows that the LO coefficient $r_{42}^{B, 0}$ starts to deviate from unity significantly for $T>150 \mathrm{MeV}$. Nonetheless, as can be seen from the inset in Fig. 1 (right), the difference of the LO expansion coefficients, $r_{42}^{B, 0}-r_{31}^{B, 0}$, stays small also in the strangeness neutral case with $n_{Q} / n_{B}=0.4$. The maximal difference is reached at $T \simeq 200 \mathrm{MeV}$ where it amounts to about $12 \%$ of $r_{31}^{B, 0}$. However, in the crossover region, $145 \mathrm{MeV}<T<165 \mathrm{MeV}$, which also is the temperature range of interest for comparison with experimental data, this difference never exceeds more than $4 \%$ of $r_{31}^{B, 0}$. The experimental observation that $S_{P} \sigma_{P}^{3} / M_{P}$ and $\kappa_{P} \sigma_{P}^{2}$ tend to agree at large $\sqrt{s_{N N}}$, although they differ from unity, thus is in accordance with the QCD result,

$$
S_{B} \sigma_{B}^{3} / M_{B} \simeq \kappa_{B} \sigma_{B}^{2} \quad \text { for } R_{12}^{B} \rightarrow 0 .
$$

\section{NEXT-TO-LEADING ORDER EXPANSIONS OF CUMULANT RATIOS}

The NLO corrections in the series expansion of ratios $R_{n m}^{B}$ at fixed temperature as well as on lines in the $T-\mu_{B}$ plane have been introduced in Eqs. (9)-(11) and in Eqs. (14)-(16), respectively. We will derive the NLO expansion coefficients in the following and show results for strangeness neutral systems with an electric charge to baryon-number ratio $n_{Q} / n_{B}=0.4$. However, for the discussion presented in this section we will also use the simpler expressions obtained for the case of vanishing strangeness and electric charge chemical potentials. In this case the information contained in the NLO expansion coefficients is much more transparent, and, as we will 
see, they show the same qualitative features and furthermore yield similar quantitative results.

The NLO expansions for cumulants and the resulting expansions of cumulant ratios for arbitrary values of the chemical potentials $\vec{\mu}=\left(\mu_{B}, \mu_{Q}, \mu_{S}\right)$ are given in the Appendix. From these one easily obtains the NLO expansion coefficients $r_{12}^{B, 3}, r_{31}^{B, 2}$, and $r_{42}^{B, 2}$ for $\mu_{Q}=\mu_{S}=0$ by evaluating these expressions for $s_{i}=q_{i}=0$ for $i=1,3$. This yields for the ratio of NLO and LO expansion coefficients,

$$
\begin{gathered}
\frac{r_{12}^{B, 3}}{r_{12}^{B, 1}}=-\frac{1}{3} \frac{\chi_{4}^{B}}{\chi_{2}^{B}}, \\
\frac{r_{31}^{B, 2}}{r_{31}^{B, 0}}=\frac{1}{6}\left(\frac{\chi_{6}^{B}}{\chi_{4}^{B}}-\frac{\chi_{4}^{B}}{\chi_{2}^{B}}\right), \\
\frac{r_{42}^{B, 2}}{r_{42}^{B, 0}}=3 \frac{r_{31}^{B, 2}}{r_{31}^{B, 0}} .
\end{gathered}
$$

As the quadratic and quartic cumulants of net baryonnumber fluctuations are positive for all values of the temperature [25], the NLO expansion coefficient of $R_{12}^{B}=M_{B} / \sigma_{B}^{2}$ is negative for all $T$. The NLO expansion coefficient of $R_{31}^{B}=S_{B} \sigma_{B}^{3} / M_{B}$ is negative as long as $\chi_{6}^{B} / \chi_{4}^{B}<\chi_{4}^{B} / \chi_{2}^{B}$. As known from the Taylor expansion of the equation of state (Fig. 13 of Ref. [25]) this is the case, at least for $T \gtrsim 155 \mathrm{MeV}$. Furthermore, Eq. (28) explicitly states that the NLO correction to the kurtosis ratio $R_{42}^{B}$ is three times larger than that for the skewness ratio $R_{31}^{B}$ for all $T$ as long as $\mu_{Q}=\mu_{S}=0$.

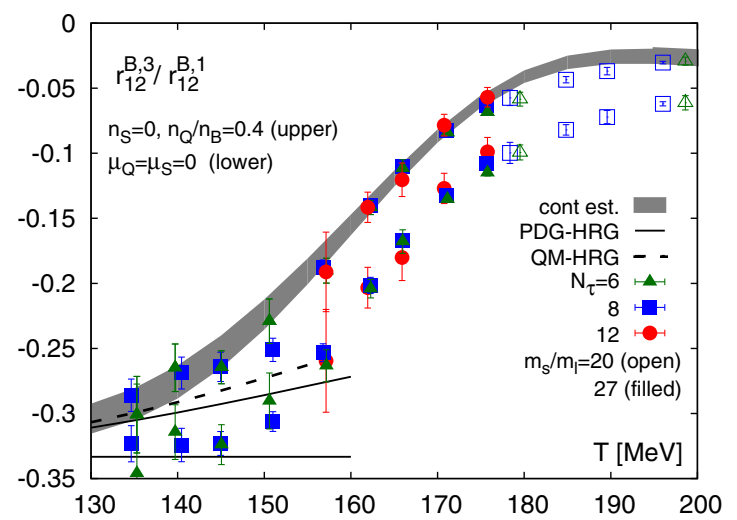

Using Eq. (26) and Eq. (27), it also is straightforward to obtain the NLO expansion coefficient of $R_{32}^{B} \equiv S_{B} \sigma_{B}$,

$$
\frac{r_{32}^{B, 3}}{r_{32}^{B, 1}}=\frac{r_{31}^{B, 2}}{r_{31}^{B, 0}}+\frac{r_{12}^{B, 3}}{r_{12}^{B, 1}}=\frac{1}{6} \frac{\chi_{6}^{B}}{\chi_{4}^{B}}-\frac{1}{2} \frac{\chi_{4}^{B}}{\chi_{2}^{B}},
$$

which also is negative, at least for $T \gtrsim 155 \mathrm{MeV}$ (see Fig. 13 of Ref. [25]).

\section{A. NLO expansion coefficients of $\boldsymbol{R}_{12}^{B}$}

In Fig. 2 we show results for the ratio of NLO and LO expansion coefficients of $R_{12}^{B}$. The left-hand figure shows the ratio of expansion coefficients for a Taylor series evaluated at fixed temperature for the two cases (i) $\mu_{S}=\mu_{Q}=0$ and (ii) $n_{S}=0, n_{Q} / n_{B}=0.4$. It is obvious that the simpler case (i) is qualitatively similar to the strangeness neutral case (ii). However, in the latter case the ratio of NLO and LO expansion coefficients is systematically smaller in magnitude.

In order to judge the importance of additional contributions to NLO expansion coefficients that arise from the variation of $T$ along a line $T_{f}\left(\mu_{B}\right)$ in the $T-\mu_{B}$ plane, we use the parametrization given in Eq. (12). We are particularly interested in a line that characterizes the change of freezeout conditions that results from changes of the beam energy in heavy ion collisions experiments. Of course, such a line eventually needs to be determined from the experimental data. However, it has been suggested $[31,42]$ that hadronic freeze-out in heavy ion collisions may take place along a line on which some thermodynamic observables stay constant as functions of $\left(T, \mu_{B}\right)$. Such "lines of constant physics" have been determined from the Taylor expansions of bulk thermodynamic observables [25] up to $\mathcal{O}\left(\mu_{B}^{4}\right)$.

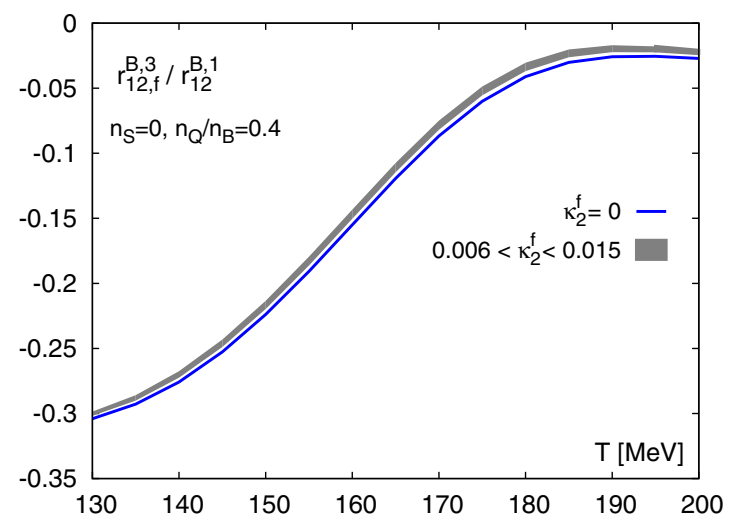

FIG. 2. Ratio of NLO and LO expansion coefficients of the cumulant ratio $R_{12}^{B} \equiv M_{B} / \sigma_{B}^{2}$ versus temperature. The left-hand figure shows results for the NLO expansion coefficient evaluated at fixed temperature while the right-hand figure gives results for the NLO expansion coefficient on a line in the $T-\mu_{B}$ plane defined in Eq. (12). The lower data set in the left-hand figure corresponds to the case $\mu_{Q}=\mu_{S}=0$ and the upper data set corresponds to the strangeness neutral case with $n_{Q} / n_{B}=0.4$. See Sec. IV for a discussion of the HRG model curves shown in the left-hand figure. The right-hand figure shows fits to the ratio $r_{12, f}^{B, 3} / r_{12}^{B, 1}$ in the strangeness neutral case for expansion coefficients at fixed temperature, i.e. for $\kappa_{f}=0$ (lower line) and on lines, $T_{f}\left(\mu_{B}\right)$, with curvature coefficients in the range $0.006 \leq \kappa_{2}^{f} \leq 0.015$. For clarity no error band is shown in this figure. 

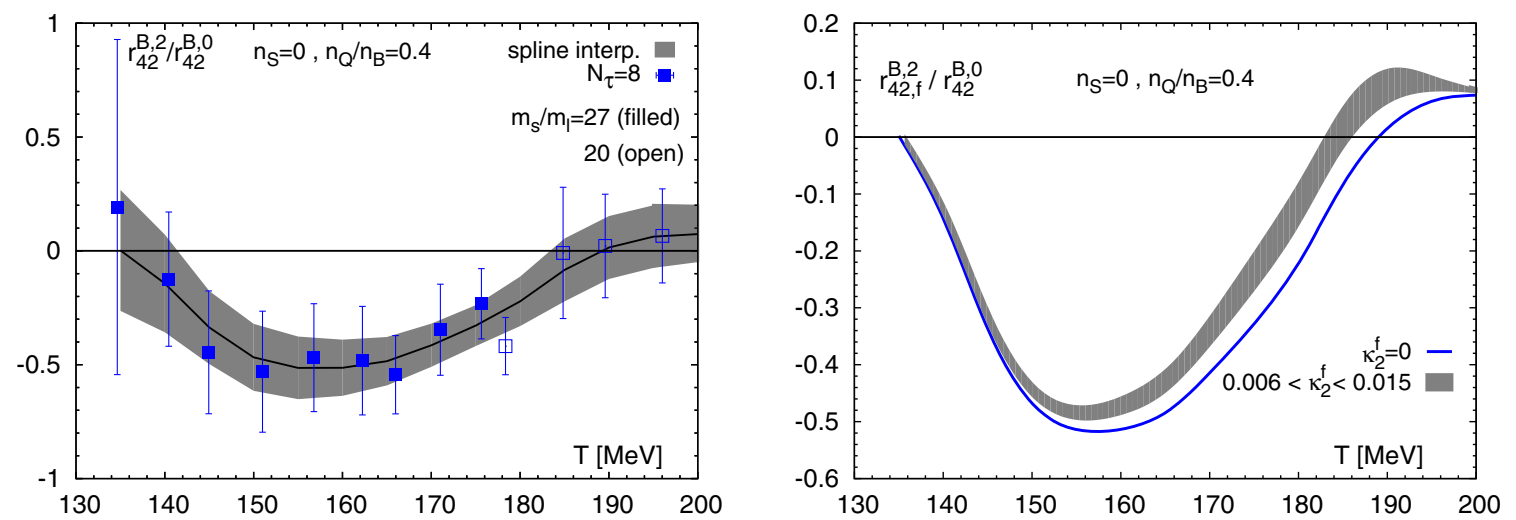

FIG. 3. Left: The ratio of NLO and LO expansion coefficients, $r_{42}^{B, 2} / r_{42}^{B, 0}$, versus temperature for a strangeness neutral system, $n_{S}=0$, with electric charge to baryon-number ratio $n_{Q} / n_{B}=0.4$. The black line shows the central value which is identical to the solid line for the $\kappa_{2}^{f}=0$ case shown in the right and figure. Right: The ratio $r_{42, f}^{B, 2} / r_{42}^{B, 0}$, which gives the ratio of NLO and LO expansion coefficients evaluated on a line in the $T-\mu_{B}$ plane as defined in Eq. (12). The band shows the shift of this ratio (central values only) resulting from a variation of $\kappa_{2}^{f}$ in the indicated interval.

For the purpose of our current NLO analysis it suffices to use information from these expansions that defines the lines $T_{f}\left(\mu_{B}\right)$ up to $\mathcal{O}\left(\mu_{B}^{2}\right)$. It turns out that lines of constant pressure, energy density, or entropy density describe similar trajectories in the $T-\mu_{B}$ plane. At NLO such lines are controlled by the curvature coefficient $\kappa_{2}^{P}$ (pressure), $\kappa_{2}^{\epsilon}$ (energy density), or $\kappa_{2}^{s}$ (entropy density), respectively. In the crossover region, $T_{c}=(154 \pm 9) \mathrm{MeV}$, we find that these curvature coefficients vary in the range, ${ }^{2}$

$$
0.006 \leq \kappa_{2}^{f} \leq 0.012, \quad f=P, \epsilon, s
$$

For baryon chemical potentials $\mu_{B} / T \leq 1.5$ the temperature variation on a line $T_{f}\left(\mu_{B}\right)$ with $\kappa_{2}^{f} \leq 0.012$ thus is less than $3 \%$ of the $T$-value at $\mu_{B}=0$. The $\mu_{B}$-dependence of the chiral crossover transition [34-37] is similar in magnitude. This range of curvature coefficients also is consistent with the bound on $\kappa_{2}^{f}$ extracted in [38] by comparing experimental data for $M_{P} / \sigma_{P}^{2}$ and the corresponding electric charge ratio $M_{Q} / \sigma_{Q}^{2}$ with a NLO lattice QCD calculation.

The right-hand part of Fig. 2 shows the influence of a nonvanishing curvature coefficient, $\kappa_{2}^{f} \leq 0.015$, on the NLO expansion coefficients for $R_{12}^{B} \equiv M_{B} / \sigma_{B}^{2}$. As can be seen, the modification is small, leading at most to a $10 \%$ change of the NLO expansion coefficient in the crossover region.

\section{B. NLO expansion coefficients of $R_{42}^{B}$ and $R_{31}^{B}$}

The ratio of NLO and LO expansion coefficients for the kurtosis ratio $R_{42}^{B}$ is shown in Fig. 3. The left-hand figure

\footnotetext{
${ }^{2}$ The temperature dependence of these curvature coefficients for the three different bulk thermodynamic observables is shown in Fig. 14 of Ref. [25].
}

shows results for expansion coefficients in the Taylor series evaluated at fixed temperature. Here only the high statistics lattice QCD results obtained on lattices with temporal extent $N_{\tau}=8$ are shown for the strangeness neutral case with $n_{Q} / n_{B}=0.4$. The band gives a spline interpolation of the numerical results. The central line of this interpolation is given as a black line. Although statistical errors are large for these expansion coefficients, which receive contributions from many sixth order cumulants, it is apparent that they are negative for temperatures $145 \mathrm{MeV} \lesssim T \lesssim 175 \mathrm{MeV}$.

Similar to what has been shown in Fig. 2, we show in the right-hand part of Fig. 3 the influence of a nonvanishing curvature coefficient, $\kappa_{2}^{f}$, on the NLO expansion coefficients for $R_{42}^{B} \equiv \kappa_{B} \sigma_{B}^{2}$. Also in this case the contribution arising from a nonvanishing $\kappa_{2}^{f}$ is small. Compared to the LO contribution, however, the NLO correction to $R_{42}^{B}$ is large. In the temperature range of interest for a discussion of freeze-out conditions in heavy-ion collisions, $145 \mathrm{MeV} \lesssim T \lesssim 165 \mathrm{MeV}$, the magnitude of $r_{42, f}^{B, 2}$ varies between $35 \%$ and $50 \%$ of $r_{42}^{B, 0}$.

The above observations also hold for the NLO corrections to the skewness ratio $R_{31}^{B}$. We show a comparison of $r_{42}^{B, 2}$ and three times $r_{31}^{B, 2}$ in Fig. 4 (left). Obviously, despite of the large statistical errors, the central values of these observables match quite well. This hints at a strong correlation between these two NLO expansion coefficients and allows one to determine their ratio to much better accuracy than the individual values would suggest. Nonetheless, the jackknife analysis of the ratio $r_{42}^{B, 2} / r_{31}^{B, 2}$ still is difficult at low and high temperatures where both observables are compatible with zero within errors. However, in the temperature interval $145 \mathrm{MeV}<T<$ $175 \mathrm{MeV}$, these expansion coefficients are clearly negative and errors are sufficiently small to determine 

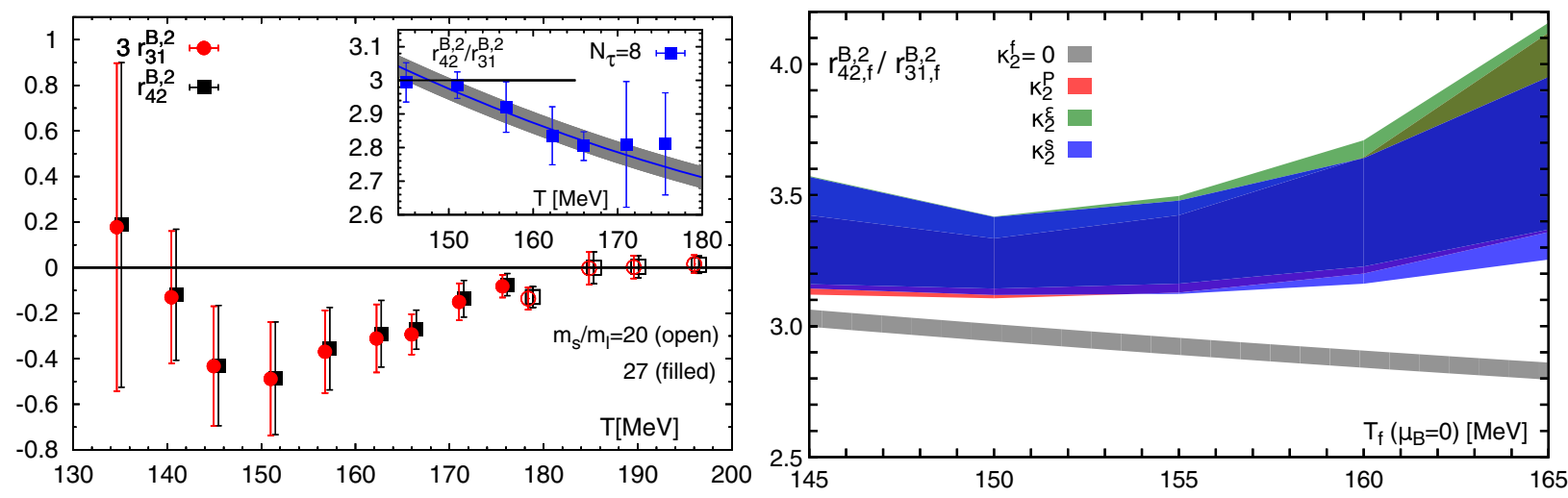

FIG. 4. Left: The NLO expansion coefficient for the kurtosis ratio, $r_{42}^{B, 2}$, and three times the NLO expansion coefficient for the skewness ratio, $r_{31}^{B, 2}$. The inset shows the ratio of the NLO expansion coefficients, $r_{42}^{B, 2} / r_{31}^{B, 2}$, in temperature range where jackknife estimators for this ratio are stable. Right: Ratio of NLO expansion coefficients of the skewness and kurtosis ratios on lines of constant physics defined by pressure, energy density, and entropy density, respectively. Also shown is the result for vanishing curvature coefficient $\left(\kappa_{2}^{f}=0\right)$. Both figures show results for a strangeness neutral system, $n_{S}=0$, with electric charge to baryon-number ratio $n_{Q} / n_{B}=0.4$.

the ratio $r_{42}^{B, 2} / r_{31}^{B, 2}$ reliably. This is shown in the inset of Fig. 4 (left). As expected, we find that also in the strangeness neutral case the ratio of expansion coefficients is close to three. This also is the case for $\mu_{Q}=\mu_{S}=0$ (see Eq. (28). The ratio has the tendency to drop with increasing temperature, suggesting that it will approach the ideal gas value at high temperature ${ }^{3}$

Using the temperature dependent curvature coefficients $\kappa_{2}^{f}$ we can determine the correction to NLO expansion coefficients of the skewness $\left(R_{31}^{B}\right)$ and kurtosis $\left(R_{42}^{B}\right)$ ratios, which arises from a $\mu_{B}$-dependent freeze-out temperature. For $\kappa_{2}^{f}=0$, the ratio, $r_{42}^{B, 2} / r_{31}^{B, 2}$, is shown as an inset in Fig. 4 (left) together with a quadratic fit. This ratio drops from 3.03(4) at $T=145 \mathrm{MeV}$ to $2.83(4)$ at $T=165 \mathrm{MeV}$. For $\kappa_{2}^{f}>0$, the ratio, $r_{42, f}^{B, 2} / r_{31, f}^{B, 2}$, will be larger than these values. This can be seen from the fact that for $\kappa_{2}^{f}>0$, the NLO coefficients $r_{42}^{B, 2}$ and $r_{31}^{B, 2}$ are shifted by almost the same positive constant (the temperature derivatives of $r_{42}^{B, 0}$ and $r_{31}^{B, 0}$ are negative and very similar in magnitude), and in the temperature range of interest both $r_{42}^{B, 2}$ and $r_{31}^{B, 2}$ are negative with $r_{42}^{B, 2} \sim 3 r_{31}^{B, 2}$. As these derivatives are small for $T \lesssim 145 \mathrm{MeV}$, and are largest for $T \simeq 165 \mathrm{MeV}$ we thus expect the difference between the ratios $r_{42, f}^{B, 2} / r_{31, f}^{B, 2}$ evaluated for $\kappa_{2}^{f}=0$ and $\kappa_{2}^{f}>0$ to rise when increasing the temperature towards the upper end of the crossover region. This is apparent from the results shown in Fig. 4 (right). Taking into account current uncertainties

\footnotetext{
${ }^{3}$ In the infinite temperature limit cumulants approach the ideal gas limit. For the ratio of NLO expansion coefficients one finds in this limit, $r_{42}^{B, 2} / r_{31}^{B, 2}=1.98$ for the strangeness neutral case, $n_{S}=0$, with $n_{Q} / n_{B}=0.4$.
}

on the coefficients $\kappa_{2}^{f}$, we find in the temperature range $145 \mathrm{MeV} \leq T \leq 165 \mathrm{MeV}$,

$$
\frac{r_{42, f}^{B, 2}}{r_{31, f}^{B, 2}}=3.1-4.1
$$

\section{COMPARING NLO LATTICE QCD CALCULATIONS WITH EXPERIMENTAL DATA}

Qualitative features of the NLO expansions for ratios of cumulants of net baryon-number fluctuations, derived in the previous sections, may be confronted with experimental results on cumulant ratios of net proton-number fluctuations. Of course, as pointed out in the introduction, one cannot directly compare the experimental data on net proton-number fluctuations with those for net baryon-number fluctuations. In particular, the systematic differences between the two sets of data [10-12] taken in different transverse momentum intervals, as well as the known sensitivity of the data on acceptance cuts, indicate that these systematic effects need to be taken care of, e.g. by implementing them in realistic hydrodynamic and transport calculations, before a quantitative comparison becomes possible. A recent study, for instance, suggests that effects of volume fluctuations are small and also effects arising from hadronic scattering and resonance decays have little influence on the ratios of net proton-number cumulants [43].

Since experimentally measured cumulants of net protonnumber fluctuations can be different from the cumulants of net baryon-number fluctuations [13], a direct comparison between the two is subject to systematic errors. However, as we will see, qualitative trends, visible in the experimental data at beam energies $\sqrt{s_{N N}} \geq 19.6 \mathrm{GeV}$, agree well with 

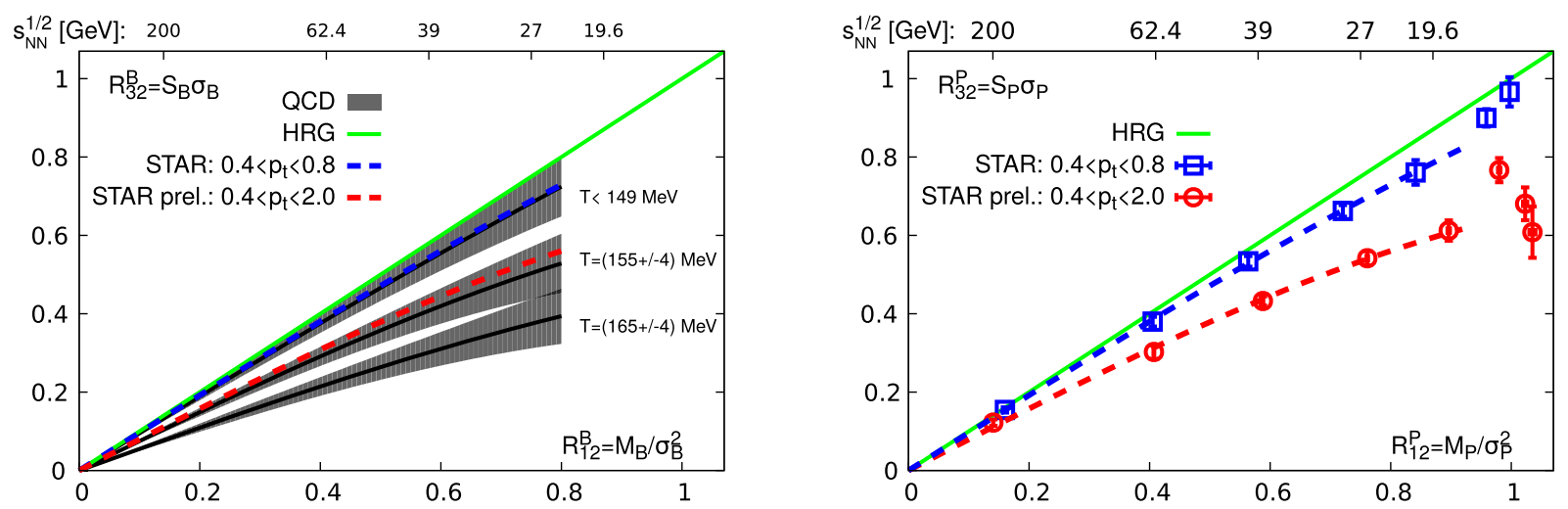

FIG. 5. Left: Next-to-leading order lattice QCD result for $S_{B} \sigma_{B}$ versus $M_{B} / \sigma_{B}^{2}$. Bands reflect the statistical errors of the lattice QCD calculation. The dashed lines show the fits to the experimental data sets shown in the right-hand figure. Right: Experimental data on $S_{P} \sigma_{P}$ versus $M_{P} / \sigma_{P}^{2}$. Shown are data obtained by the STAR collaboration in two different transverse momentum intervals and for different values of the beam energy, $\sqrt{s_{N N}} / \mathrm{GeV}=200,62.4,39,27,19.6,11.5,7.7$. For the preliminary data in the larger $p_{t}$ range an additional data point is shown at $14.5 \mathrm{GeV}$ [12]. The dashed lines show cubic fits, $a R_{12}^{P}+b\left(R_{12}^{P}\right)^{3}$, for $\sqrt{s_{N N}} \geq 19.6 \mathrm{GeV}$.

QCD results on cumulant ratios and their dependence on the baryon chemical potential. They are consistent with a freeze-out temperature close to the QCD transition temperature.

For the comparison with experimental data, we will use the expansion of higher order cumulant ratios on lines of constant physics in terms of $R_{12}^{B}$ as given in Eq. (18) to Eq. (20). This allows us to compare lattice QCD calculations with experimental data without prior determination of the chemical potential, $\mu_{B}$.

In Fig. 5 (left) we show the skewness ratio $R_{32}^{B}=S_{B} \sigma_{B}$ calculated in a NLO Taylor series. Results are shown as a function of $R_{12}^{B}$ for three temperature intervals. These temperature intervals have been fixed, somewhat arbitrarily, by choosing three intervals for the slope parameter, $r_{31}^{B, 0}$, i.e. (i) $r_{31}^{B, 0}=0.95(5)$, (ii) $r_{31}^{B, 0}=0.75(5)$, (iii) $r_{31}^{B, 0}=0.55(5)$. These intervals correspond to temperature intervals centered around the (i) higher, (ii) central, and (iii) lower value of the QCD transition range $T_{c}=154(9) \mathrm{MeV}$. Taking into account the error band on the spline interpolation shown in Fig. 1 (right), this leads to the error bands and $T$-intervals given in Fig. 5 (left).

As is obvious from the temperature dependence of $r_{42}^{B, 0}$ and $r_{31}^{B, 0}$, shown in Fig. 1 (right), the slope of $R_{32}^{B}$, which equals $r_{31}^{B, 0}$, decreases with increasing temperature and the NLO corrections lead to a bending of the curves away from the simple straight line result, which also is obtained in a HRG model calculation with noninteracting, pointlike hadrons. The central temperature range $151 \mathrm{MeV} \leq T \leq$ $159 \mathrm{MeV}$, corresponding to the central value obtained for the QCD transition temperature, also covers the freeze-out temperature range determined by the ALICE collaboration at the LHC for almost vanishing chemical potential, $T_{f}=$ 156(2) MeV [44]. This band also is consistent with cumulant ratio results obtained by the STAR collaboration from an analysis of cumulant ratios measured at mid-rapidity, $|y| \leq 0.5$, including protons and antiprotons with transverse momenta $0.4 \mathrm{GeV} \leq p_{t} \leq 2.0 \mathrm{GeV}$ $[11,12]$. These data and the corresponding STAR data set in a smaller $p_{t}$-interval, $0.4 \mathrm{GeV} \leq p_{t} \leq 0.8 \mathrm{GeV}$ [10] are shown in Fig. 5 (right). We have fitted these data for $\sqrt{s_{N N}} \geq 19.6 \mathrm{GeV}$ using a cubic ansatz, $R_{32}^{P}=S_{0} R_{12}^{P}+$ $S_{2}\left(R_{12}^{P}\right)^{3}$. The fits for the two different $p_{t}$-ranges are also shown in Fig. 5 (left). The data obtained in the larger $p_{t}$-interval are consistent with freeze-out in the vicinity of the QCD crossover temperature, while the data in the smaller $p_{t}$-interval would be consistent only with a freezeout temperature smaller than $150 \mathrm{MeV}$.

The STAR data on $R_{32}^{P}$ versus $R_{12}^{P}$, obtained at beam energies $\sqrt{s_{N N}} \geq 19.6 \mathrm{GeV}$, deviate from a linear dependence and show evidence for NLO corrections that are consistent in magnitude with those determined in the NLO lattice QCD calculation for $R_{32}^{B}$. The data obtained in the large $p_{t}$-interval are thermodynamically consistent with freeze-out happening close to the QCD transition temperature as well as a freeze-out temperature $T_{f} \simeq$ $156 \mathrm{MeV}$ as determined by the ALICE collaboration. However, these results disfavor a large freeze-out temperature, $T_{f} \simeq 165 \mathrm{MeV}$, as determined by the STAR collaboration at large beam energies [45].

The fact that the slope of $R_{32}^{B}$ differs from unity and decreases with increasing temperature is equivalent to stating that the intercept of the skewness ratio $R_{31}^{B}=$ $S_{B} \sigma_{B}^{3} / M_{B}$ at vanishing $R_{12}^{B}$ is smaller than unity and also decreases with increasing temperature. As can be seen in Eq. (18) and Eq. (20) the LO and NLO expansion coefficients of $R_{32}^{B}$ and $R_{31}^{B}$ are, of course, identical. The fits shown in Fig. 5 (right) thus also describe the experimental data on the skewness ratio shown in Fig. 6. The corresponding results for the skewness ratio of net baryonnumber fluctuations obtained from the NLO lattice QCD calculation are shown as black bands in Fig. 7 for the three 

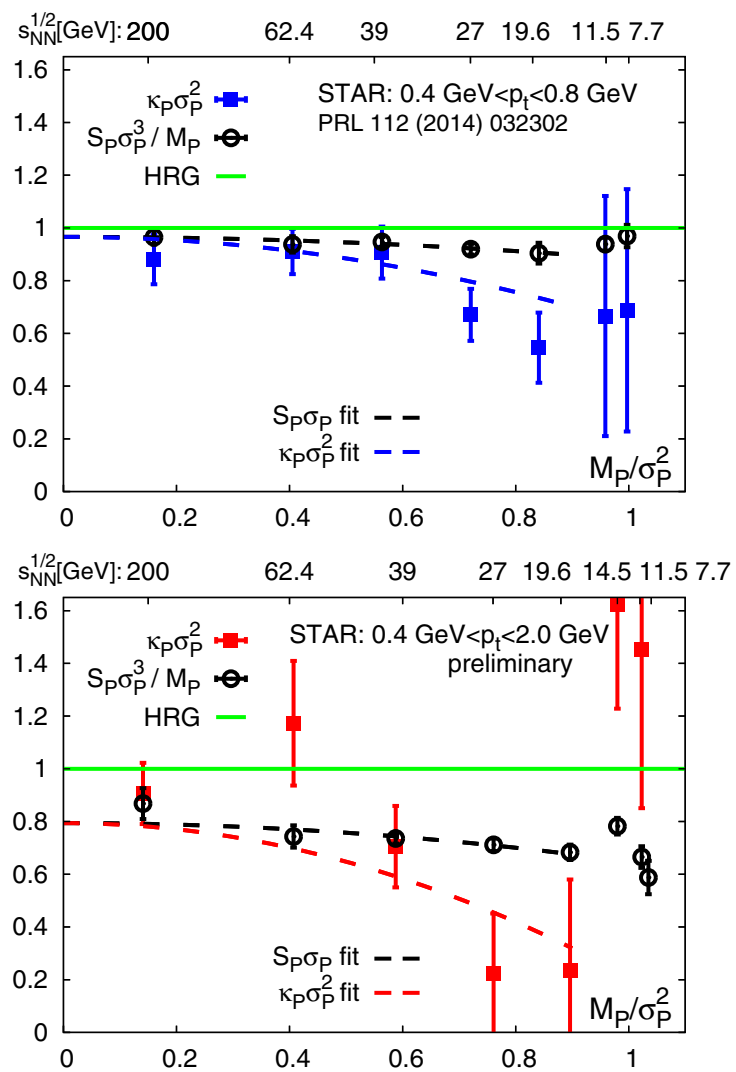

FIG. 6. Skewness and kurtosis ratios for net proton-number distributions defined in Eq. (1) versus mean net proton-number divided by variance. Data points are for different values of the beam energies as specified in Fig. 5. In the bottom figure a data point for $\kappa_{P} \sigma_{P}^{2}$ at the lowest beam energy $\sqrt{s_{N N}}=7.7 \mathrm{GeV}$ is not shown. See text for a discussion of the fits.

temperature intervals defined previously. These bands are simply obtained from those shown in Fig. 5 (left) by dividing with $R_{12}^{B}$.

The additional fits for the kurtosis ratio $R_{42}^{P}$ shown in Fig. 6 have been obtained by using the quadratic fit ansatz, $R_{42}^{P}=K_{0}+K_{2}\left(R_{12}^{P}\right)^{2}$, with $K_{0} \equiv S_{0}$. I.e., we demand that the skewness and kurtosis ratios have identical intercepts at $R_{12}^{P}=0$. These constrained fits provide a good description of the data with $K_{2}=(4 \pm 2) S_{2}$. Both fits, shown as blue and red dashed lines in Fig. 6, are also shown in Fig. 7. The ratio $K_{2} / S_{2}$ should be compared to the ratio of slope parameters, $r_{42, f}^{B, 2} / r_{31, f}^{B, 2}$, for the corresponding kurtosis and skewness ratios of net baryon-number fluctuations, which is given in Eq. (31). The trend and even the magnitude of this ratio agrees well with the experimental data. The stronger bending of the kurtosis ratio relative to the skewness ratio of net proton-number fluctuations observed experimentally thus finds a natural explanation in the NLO lattice QCD calculation.

The general pattern seen in the STAR data for $S_{P} \sigma_{P}^{3} / M_{P}$ and $\kappa_{P} \sigma_{P}^{2}$ for the two different $p_{t}$-intervals is consistent with what we have discussed for $S_{P} \sigma_{P}$ in connection with

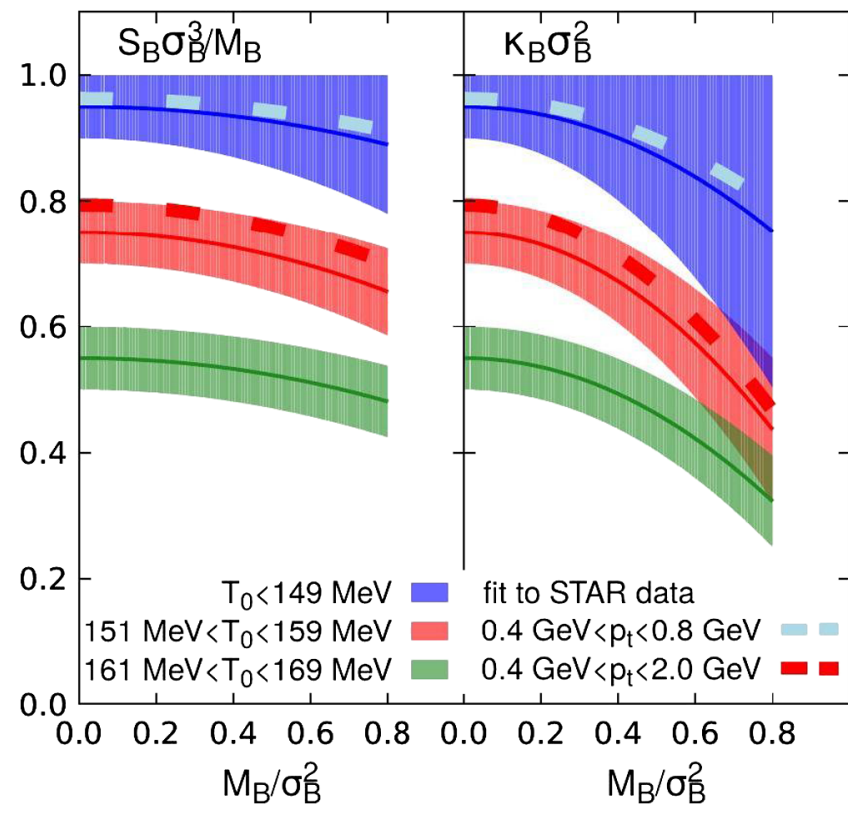

FIG. 7. NLO expansion of the skewness (left) and kurtosis (right) ratios $S_{B} \sigma_{B}^{3} / M_{B}$ and $\kappa_{B} \sigma_{B}^{2}$, respectively. Shown are results in three temperature ranges covering the crossover region of the QCD transition at vanishing baryon chemical potential. Dashed lines show the fits to experimental results for the corresponding skewness and kurtosis ratios of net proton-number fluctuations. These fits are also shown in Fig. 6.

Fig. 5. The data obtained in the large $p_{t}$-interval are consistent with a freeze-out temperature close to the QCD transition temperature, while the data obtained in the smaller $p_{t}$-interval are thermodynamically consistent only with a small freeze-out temperature, $T_{f}<150 \mathrm{MeV}$. A large freeze-out temperature of about $165 \mathrm{MeV}$, on the other hand, would require that the skewness and kurtosis ratios become substantially smaller, even at large beam energies once all potential systematic corrections have been taken into account.

The fit to the data for $R_{31}^{P}$ obtained in the large $p_{t}$-interval, which is shown in Fig. 6, gives the value $R_{31}^{P}=R_{42}^{P}=0.80(4)$ for the intercept at $R_{12}^{P}=0$. This also is shown as a gray box in Fig. 1 (right) and constrains the range of freeze-out temperatures quite well. We conclude that all current data on cumulant ratios, measured by STAR in the different transverse momentum intervals, $0.4 \mathrm{GeV}<p_{t}<p_{t}^{\text {cut }}$, are consistent with freeze-out temperatures,

$$
\begin{aligned}
& T_{0} \leq 149 \mathrm{MeV} \quad \text { for } p_{t}^{\text {cut }}=0.8 \mathrm{GeV} \\
& T_{0}=(153 \pm 5) \mathrm{MeV} \quad \text { for } p_{t}^{\text {cut }}=2.0 \mathrm{GeV}
\end{aligned}
$$

The latter is in excellent agreement with the freeze-out temperature determined by the ALICE Collaboration from particle yields at the LHC [44] but differs significantly from the freeze-out parameters at large beam energies presented 
by STAR [45]. Within errors it also is consistent with the somewhat lower value for $T_{f}$ extracted in our analysis of ratios of variances of net electric charge and net baryonnumber fluctuations [38].

Nonetheless, as stressed above, this observation can only be taken as a first indication, given the observed dependence of $S_{P} \sigma_{P}^{3} / M_{P}$ on the transverse momentum range analyzed as well as the size of rapidity bins entering the analysis.

\section{SUMMARY AND CONCLUSIONS}

Next-to-leading order calculations of cumulant ratios involving the skewness and kurtosis of net baryon-number fluctuations are computationally demanding as they involve many 6th order cumulants of conserved charges fluctuations that are difficult to calculate and statistically noisy in lattice QCD. Depending on the temperature value the analysis presented here required the generation of up to 7 million time units in RHMC simulations to control these NLO corrections on lattices of size $32^{3} \times 8$.

Most of the calculations presented here are not yet extrapolated to the continuum limit. They, however, clearly show that qualitative features of currently available experimental data on net proton-number cumulants can be understood in terms of equilibrium thermodynamics of QCD. In the range of applicability, $\mu_{B} \lesssim 200 \mathrm{MeV}$, which corresponds to energies $\sqrt{s_{N N}} \gtrsim 19 \mathrm{GeV}$ in the RHIC beam energy scan, the QCD-based calculations presented here may explain

(i) the deviation of $S_{P} \sigma_{P}^{3} / M_{P}$ from unity,

(ii) the coincidence of the skewness ratio $S_{P} \sigma_{P}^{3} / M_{P}$ and the kurtosis ratio $\kappa_{P} \sigma_{P}^{2}$ for large RHIC beam energy, $\sqrt{s_{N N}} \gtrsim 200 \mathrm{GeV}$,

(iii) the significantly stronger decrease of $\kappa_{P} \sigma_{P}^{2}$, in comparison to $S_{P} \sigma_{P}^{3} / M_{P}$, with decreasing beam energies down to $\sqrt{s_{N N}}=19.6 \mathrm{GeV}$.

We have shown that NLO corrections to the skewness and kurtosis ratios, evaluated for the strangeness neutral case, are negative in the entire interval $145 \mathrm{MeV} \lesssim T \lesssim 175 \mathrm{MeV}$. This also holds for the case $\mu_{Q}=\mu_{S}=0$. As shown in Eq. (27) and Eq. (28), it is evident that negative values for the skewness and kurtosis ratios in this case imply $\chi_{6}^{B} / \chi_{4}^{B}<\chi_{4}^{B} / \chi_{2}^{B}$. In fact, the 6th order cumulant of net baryon-number fluctuations turns out to be negative in this temperature range.

It is conceivable that higher order cumulants will start changing signs in an irregular pattern for $T \gtrsim 145 \mathrm{MeV}$, indicating that the radius of convergence of the Taylor series for the QCD pressure is limited by a singularity in the complex plane (strictly alternating signs of expansion coefficients would correspond to a singularity for purely imaginary values of $\mu_{B} / T$ ). Such a scenario disfavors the location of a critical point in the QCD phase diagram for $T \gtrsim 145 \mathrm{MeV}$.
The observation that Taylor series for skewness and kurtosis of net baryon-number fluctuations closely resemble the corresponding experimental results for the net proton-number fluctuations for $\mu_{B} \leq 200 \mathrm{MeV}$ is, thus, consistent with the analysis of the radius of convergence of the Taylor series for the pressure and second order net baryon-number cumulants [25], which leads to the conclusion that a critical point at $\mu_{B} / T \leq 2$ is disfavored by current lattice QCD calculations.

\section{ACKNOWLEDGMENTS}

This work was supported in part through Contract No. DE-SC001270 with the U.S. Department of Energy, through the Scientific Discovery through Advanced Computing (SciDAC) program funded by the U.S. Department of Energy, Office of Science, Advanced Scientific Computing Research and Nuclear Physics, the DOE Office of Nuclear Physics funded BEST topical collaboration and the NERSC Exascale Application Program (NESAP). This work was also performed under the auspices of the U.S. Department of Energy by Lawrence Livermore National Laboratory under Contract DE-AC52-07NA27344. Furthermore, this work was supported by the Deutsche Forschungsgemeinschaft (DFG) through the Grant No. CRC-TR 211 "Stronginteraction matter under extreme conditions", the Grant No. 05P15PBCAA of the German Bundesministerium für Bildung und Forschung, Grant No. 283286 of the European Union, the National Natural Science Foundation of China under Grant No. 11535012 and No. 11521064, and the Early Career Research Award of the Science and Engineering Research Board of the Government of India. Numerical calculations have been made possible through an INCITE grant of USQCD, ALCC grants in 2016 and 2017, and PRACE grants at CINECA, Italy. These grants provided access to resources on Titan at ORNL, BlueGene/Q at ALCF, Cori-II at NERSC, and Marconi at CINECA. Additional numerical calculations have been performed on USQCD GPU and KNL clusters at JLab and Fermilab, as well as GPU clusters at Bielefeld University and Paderborn University. We thank the Lawrence Livermore National Laboratory (LLNL) Multiprogrammatic and Institutional Computing program for Grand Challenge allocations and time on the LLNL BlueGene/Q supercomputer. We furthermore acknowledge the support of NVIDIA through the CUDA Research Center at Bielefeld University.

\section{APPENDIX: NLO EXPANSION COEFFICIENTS FOR $\chi_{n}^{B}$}

We give here the next-to-leading order results for the expansion coefficients of up to fourth order cumulants of net baryon-number fluctuations in the constrained case, where $\mu_{Q}$ and $\mu_{S}$ get replaced by Eq. (6). The expansions of 
even and odd order cumulants in terms of the baryon chemical potential, $\hat{\mu}_{B}=\mu_{B} / T$, are given by

$$
\begin{gathered}
\chi_{2 n}^{B}\left(T, \mu_{B}\right)=\bar{\chi}_{2 n}^{B, 0}+\frac{1}{2} \bar{\chi}_{2 n}^{B, 2} \hat{\mu}_{B}^{2}, \quad n=1,2 \\
\chi_{2 n+1}^{B}\left(T, \mu_{B}\right)=\bar{\chi}_{2 n+1}^{B, 1} \hat{\mu}_{B}+\frac{1}{6} \bar{\chi}_{2 n+1}^{B, 3} \hat{\mu}_{B}^{3}, \quad n=0,1 .
\end{gathered}
$$

Here the $k$-th order expansion coefficients $\bar{\chi}_{n}^{B, k}$ are functions of temperature and the Taylor expansion coefficients $q_{i}, s_{i}$ of the electric charge and strangeness chemical potentials (see Eq. (6). The LO expansion coefficients are given by,

$$
\begin{aligned}
& \bar{\chi}_{1}^{B, 1}=\chi_{2}^{B}+s_{1} \chi_{11}^{B S}+q_{1} \chi_{11}^{B Q}, \\
& \bar{\chi}_{2}^{B, 0}=\chi_{2}^{B}, \\
& \bar{\chi}_{3}^{B, 1}=\chi_{4}^{B}+s_{1} \chi_{31}^{B S}+q_{1} \chi_{31}^{B Q}, \\
& \bar{\chi}_{4}^{B, 0}=\chi_{4}^{B} .
\end{aligned}
$$

We note that in LO the expansion coefficients for even order cumulants do not depend on the constraint put on strangeness and electric charge densities, while the odd order expansion coefficients explicitly depend on them. The NLO expansion coefficients for odd order cumulants, $\bar{\chi}_{n}^{B, 3}, n=1,3$, can be written as,

$$
\bar{\chi}_{n}^{B, 3}=m_{n}^{B, 3}+6 s_{3} \chi_{n 1}^{B S}+6 q_{3} \chi_{n 1}^{B Q},
$$

with

$$
\begin{aligned}
m_{1}^{B, 3}= & \chi_{4}^{B}+\chi_{13}^{B S} s_{1}^{3}+\chi_{13}^{B Q} q_{1}^{3}+3 \chi_{22}^{B S} s_{1}^{2}+3 \chi_{22}^{B Q} q_{1}^{2} \\
& +3 \chi_{31}^{B S} s_{1}+3 \chi_{31}^{B Q} q_{1}+6 \chi_{211}^{B Q S} q_{1} s_{1} \\
& +3 \chi_{121}^{B Q S} q_{1}^{2} s_{1}+3 \chi_{112}^{B Q S} q_{1} s_{1}^{2},
\end{aligned}
$$

and

$$
\begin{aligned}
m_{3}^{B, 3}= & \chi_{6}^{B}+\chi_{33}^{B S} s_{1}^{3}+\chi_{33}^{B Q} q_{1}^{3}+3 \chi_{42}^{B S} s_{1}^{2}+3 \chi_{42}^{B Q} q_{1}^{2} \\
& +3 \chi_{51}^{B S} s_{1}+3 \chi_{51}^{B Q} q_{1}+6 \chi_{411}^{B Q S} q_{1} s_{1} \\
& +3 \chi_{321}^{B Q S} q_{1}^{2} s_{1}+3 \chi_{312}^{B Q S} q_{1} s_{1}^{2} .
\end{aligned}
$$

Explicit expressions for the NLO expansion coefficients $q_{3}$, $s_{3}$ of the electric charge and strangeness chemical potentials, needed in Eq. (A3), have been given in Appendix B of Ref. [25]. Similarly, the NLO expansion coefficients of even order cumulants, $\bar{\chi}_{n}^{B}, n=2,4$ are obtained as

$$
\begin{aligned}
\bar{\chi}_{2}^{B, 2}= & \chi_{4}^{B}+s_{1}^{2} \chi_{22}^{B S}+q_{1}^{2} \chi_{22}^{B Q}+2 s_{1} \chi_{31}^{B S} \\
& +2 q_{1} \chi_{31}^{B Q}+2 q_{1} s_{1} \chi_{211}^{B Q S}, \\
\bar{\chi}_{4}^{B, 2}= & \chi_{6}^{B}+s_{1}^{2} \chi_{42}^{B S}+q_{1}^{2} \chi_{42}^{B Q}+2 s_{1} \chi_{51}^{B S} \\
& +2 q_{1} \chi_{51}^{B Q}+2 q_{1} s_{1} \chi_{411}^{B Q S} .
\end{aligned}
$$

This gives for the ratios of $\mathrm{LO}$ expansion coefficients introduced in Eqs. (9)-(11),

$$
r_{12}^{B, 1}=\frac{\bar{\chi}_{1}^{B, 1}}{\bar{\chi}_{2}^{B, 0}}, \quad r_{31}^{B, 0}=\frac{\bar{\chi}_{3}^{B, 1}}{\bar{\chi}_{1}^{B, 1}}, \quad r_{42}^{B, 0}=\frac{\bar{\chi}_{4}^{B, 0}}{\bar{\chi}_{2}^{B, 0}}
$$

For the NLO expansion coefficients introduced in Eqs. (9)-(11), one then obtains,

$$
\frac{r_{12}^{B, 3}}{r_{12}^{B, 1}}=\frac{1 \bar{\chi}_{1}^{B, 3}}{6} \frac{1}{\bar{\chi}_{1}^{B, 1}}-\frac{\bar{\chi}_{2}^{B, 2}}{2} \frac{\bar{\chi}_{2}^{B, 0}}{,}
$$

$$
\frac{r_{31}^{B, 2}}{r_{31}^{B, 0}}=\frac{1}{6}\left(\frac{\bar{\chi}_{3}^{B, 3}}{\bar{\chi}_{3}^{B, 1}}-\frac{\bar{\chi}_{1}^{B, 3}}{\bar{\chi}_{1}^{B, 1}}\right)
$$

$$
\frac{r_{42}^{B, 2}}{r_{42}^{B, 0}}=\frac{1}{2}\left(\frac{\bar{\chi}_{4}^{B, 2}}{\bar{\chi}_{4}^{B, 0}}-\frac{\bar{\chi}_{2}^{B, 2}}{\bar{\chi}_{2}^{B, 0}}\right)
$$

The corresponding expansion coefficients in the case $\mu_{Q}=\mu_{S}=0$ are obtained from these expressions simply by substituting $\bar{\chi} \rightarrow \chi$.
[1] Y. Hatta and M. A. Stephanov, Phys. Rev. Lett. 91, 102003 (2003).

[2] S. Ejiri, F. Karsch, and K. Redlich, Phys. Lett. B 633, 275 (2006).

[3] V. Koch, A. Majumder, and J. Randrup, Phys. Rev. Lett. 95, 182301 (2005).
[4] H. T. Ding, F. Karsch, and S. Mukherjee, Int. J. Mod. Phys. E 24, 1530007 (2015).

[5] M. Asakawa and K. Yazaki, Nucl. Phys. A504, 668 (1989).

[6] A. M. Halasz, A. D. Jackson, R. E. Shrock, M. A. Stephanov, and J. J. M. Verbaarschot, Phys. Rev. D 58, 
096007 (1998); J. Berges and K. Rajagopal, Nucl. Phys. B538, 215 (1999).

[7] X. Luo and N. Xu, Nucl. Sci. Tech. 28, 112 (2017).

[8] L. Adamczyk et al. (STAR Collaboration), Phys. Rev. Lett. 113, 092301 (2014).

[9] A. Adare et al. (PHENIX Collaboration), Phys. Rev. C 93, 011901 (2016).

[10] M. M. Aggarwal et al. (STAR Collaboration), Phys. Rev. Lett. 105, 022302 (2010).

[11] X. Luo et al. (STAR Collaboration), Proc. Sci., CPOD2014 (2015) 019 [arXiv:1503.02558].

[12] J. Thäder et al. (STAR Collaboration), Nucl. Phys. A956, 320 (2016).

[13] M. Kitazawa and M. Asakawa, Phys. Rev. C 86, 024904 (2012); 86, 069902(E) (2012).

[14] A. Bzdak and V. Koch, Phys. Rev. C 86, 044904 (2012).

[15] B. Ling and M. A. Stephanov, Phys. Rev. C 93, 034915 (2016).

[16] A. Bzdak and V. Koch, Phys. Rev. C 91, 027901 (2015).

[17] F. Karsch, K. Morita, and K. Redlich, Phys. Rev. C 93, 034907 (2016).

[18] A. Bzdak and V. Koch, arXiv:1707.02640.

[19] B. Berdnikov and K. Rajagopal, Phys. Rev. D 61, 105017 (2000).

[20] M. Kitazawa, M. Asakawa, and H. Ono, Phys. Lett. B 728, 386 (2014).

[21] S. Mukherjee, R. Venugopalan, and Y. Yin, Phys. Rev. C 92, 034912 (2015).

[22] S. Mukherjee, R. Venugopalan, and Y. Yin, Phys. Rev. Lett. 117, 222301 (2016).

[23] M. Asakawa and M. Kitazawa, Prog. Part. Nucl. Phys. 90, 299 (2016).

[24] R. V. Gavai and S. Gupta, Phys. Lett. B 696, 459 (2011).

[25] A. Bazavov, H.-T. Ding, P. Hegde, O. Kaczmarek, F. Karsch, E. Laermann, Y. Maezawa, S. Mukherjee, H. Ohno, P. Petreczky, H. Sandmeyer, P. Steinbrecher, C. Schmidt, S. Sharma, W. Soeldner, and M. Wagner, Phys. Rev. D 95, 054504 (2017).

[26] A. Bazavov, T. Bhattacharya, M. Cheng, C. DeTar, H.-T. Ding, S. Gottlieb, R. Gupta, P. Hegde, U. M. Heller, F. Karsch, E. Laermann, L. Levkova, S. Mukherjee, P. Petreczky, C. Schmidt, R. A. Soltz, W. Soeldner, R. Sugar, D. Toussaint, W. Unger, and P. Vranas, Phys. Rev. D 85, 054503 (2012).

[27] F. Karsch, Central Eur. J. Phys. 10, 1234 (2012).
[28] A. Bazavov, H. T. Ding, P. Hegde, O. Kaczmarek, F. Karsch, E. Laermann, S. Mukherjee, P. Petreczky, C. Schmidt, D. Smith, W. Soeldner, and M. Wagner, Phys. Rev. Lett. 109, 192302 (2012).

[29] S. Mukherjee and M. Wagner, Proc. Sci., CPOD2013 (2013) 039 [arXiv:1307.6255].

[30] S. Borsanyi, Z. Fodor, S. D. Katz, S. Krieg, C. Ratti, and K. K. Szabo, Phys. Rev. Lett. 111, 062005 (2013).

[31] J. Cleymans, H. Oeschler, K. Redlich, and S. Wheaton, Phys. Rev. C 73, 034905 (2006).

[32] A. Andronic, P. Braun-Munzinger, and J. Stachel, Nucl. Phys. A772, 167 (2006).

[33] P. Braun-Munzinger, K. Redlich, and J. Stachel, in Quark Gluon Plasma 3, edited by R. C. Hwa and X. N. Wang (World Scientific, Singapore, 2004), p. 491-599.

[34] O. Kaczmarek, F. Karsch, E. Laermann, C. Miao, S. Mukherjee, P. Petreczky, C. Schmidt, W. Soeldner, and W. Unger, Phys. Rev. D 83, 014504 (2011).

[35] C. Bonati, M. D’Elia, M. Mariti, M. Mesiti, F. Negro, and F. Sanfilippo, Phys. Rev. D 92, 054503 (2015).

[36] R. Bellwied, S. Borsanyi, Z. Fodor, J. Günther, S. D. Katz, C. Ratti, and K. K. Szabo, Phys. Lett. B 751, 559 (2015).

[37] P. Cea, L. Cosmai, and A. Papa, Phys. Rev. D 93, 014507 (2016).

[38] A. Bazavov, H.-T. Ding, P. Hegde, O. Kaczmarek, F. Karsch, E. Laermann, S. Mukherjee, H. Ohno, P. Petreczky, C.Schmidt, S. Sharma, W. Soeldner, and M. Wagner, Phys. Rev. D 93, 014512 (2016).

[39] A. Bazavov, T. Bhattacharya, C. E. DeTar, H.-T. Ding, S. Gottlieb, R. Gupta, P. Hegde, U. Heller, F. Karsch, E. Laermann, L. Levkova, S. Mukherjee, P. Petreczky, C. Schmidt, C. Schroeder, R. A. Soltz, W. Soeldner, R. Sugar, M. Wagner, and P. M. Vranas (HotQCD Collaboration), Phys. Rev. D 90, 094503 (2014).

[40] A. Bazavov, H.-T. Ding, P. Hegde, O. Kaczmarek, F. Karsch, E. Laermann, Y. Maezawa, S. Mukherjee, H. Ohno, P. Petreczky, C. Schmidt, S. Sharma, W. Soeldner, and M. Wagner, Phys. Rev. Lett. 113, 072001 (2014).

[41] J. Beringer et al. (Particle Data Group), Phys. Rev. D 86, 010001 (2012).

[42] P. Castorina, A. Iorio, and H. Satz, Int. J. Mod. Phys. E 24, 1550056 (2015).

[43] J. Li, H. j. Xu, and H. Song, arXiv:1707.09742.

[44] M. Floris, Nucl. Phys. A931, 103 (2014).

[45] L. Adamczyk et al. (STAR Collaboration), arXiv: 1701.07065 [Phys. Rev. C (to be published)]. 